\title{
Relativistic attosecond physics ${ }^{\text {a) }}$
}

\author{
Natalia M. Naumova, ${ }^{\text {b) }}$ John A. Nees, and Gérard A. Mourou \\ Center for Ultrafast Optical Science and FOCUS Center, University of Michigan, \\ Ann Arbor, Michigan 48109
}

(Received 12 November 2004; accepted 14 December 2004; published online 13 April 2005)

A study, with particle-in-cell simulations, of relativistic nonlinear optics in the regime of tight focus and ultrashort pulse duration (the $\lambda^{3}$ regime) reveals that synchronized attosecond electromagnetic pulses [N. M. Naumova, J. A. Nees, I. V. Sokolov, B. Hou, and G. A. Mourou, Phys. Rev. Lett. 92, 063902 (2004)] and attosecond electron bunches [N. Naumova, I. Sokolov, J. Nees, A. Maksimchuk, V. Yanovsky, and G. Mourou, Phys. Rev. Lett. 93, 195003 (2004)] emerge efficiently from laser interaction with overdense plasmas. The $\lambda^{3}$ concept enables a more basic understanding and a more practical implementation of these phenomena because it provides spatial and temporal isolation. The synchronous generation of strong attosecond electromagnetic pulses and dense attosecond electron bunches provides a basis for relativistic attosecond optoelectronics. (C) 2005 American Institute of Physics. [DOI: 10.1063/1.1880032]

\section{INTRODUCTION}

The possibility of eventually generating electromagnetic fields capable of distorting or breaking down vacuum near the quantum electrodynamics limit is one factor drawing science toward extreme intensities. ${ }^{1}$ Current technology allows the construction of petawatt laser systems with focal intensities of $10^{23} \mathrm{~W} / \mathrm{cm}^{2}$. By focusing $0.8 \mu \mathrm{m}, 30 \mathrm{fs}$ laser pulses to a 1- $\lambda$ diameter $\operatorname{spot}^{2}$ an intensity of $10^{22} \mathrm{~W} / \mathrm{cm}^{2}$ has now been demonstrated. ${ }^{3}$ The further development of highly focusable laser systems toward exawatt and zetawatt levels ${ }^{4}$ is potentially achievable but prohibitively expensive.

Relativistic nonlinear optical effects ${ }^{5,6}$ should have the strength to enable a more practical path to higher intensities. These effects are based on the relativistic velocity of electrons, with $\gamma=\left(1-v^{2} / c^{2}\right)^{-1 / 2}$, driven by light with field strength $a_{0}=e E_{0} / m_{e} \omega_{0} c>1$ (i.e., with intensity where $I \lambda^{2}$ $>1.37 \times 10^{18} \mathrm{~W} \mathrm{~cm}^{-2} \mu \mathrm{m}^{2}$ ). At these intensities matter is transformed by light into highly ionized plasma. Laser pulses interacting with the plasma are then capable of efficiently producing electromagnetic radiation patterns and dense electron bunches each with attosecond structure. ${ }^{7-9}$ These attosecond structures then can be useful for producing higher intensities. On one hand, electromagnetic radiation of attosecond duration may be focused to a smaller volume, providing higher intensity. While, on the other hand, attosecond electron bunches may be used to coherently up-convert counterpropagating radiation which can also be focused to a minute volume.

\section{ATTOSECOND PHENOMENA IN LASER-PLASMA INTERACTIONS}

In relativistic overdense plasmas $\left[\omega_{0}<\omega_{p}, \omega_{p}\right.$ $\left.=\left(4 \pi n_{e} e^{2} / \gamma m_{e}\right)^{1 / 2}\right]$ a framework for attosecond pulse dura-

\footnotetext{
a) Paper FI1B 1, Bull. Am. Phys. Soc. 49, 134 (2004).

${ }^{b}$ Invited speaker. Electronic mail: naumova@umich.edu; URL:http:// www.eecs.umich.edu/CUOS/attosecond
}

tions was established in experiments on harmonic generation from solid targets. ${ }^{10-13}$ An oscillating mirror model, ${ }^{14,15}$ explaining harmonic generation from relativistically driven overdense plasmas, was developed for plane-wave incidence and captured the basic features of interaction.

Based on the oscillating mirror model at normal incidence a suggestion was made to generate attosecond pulse trains in the reflection of intense laser pulses from solid surfaces. ${ }^{16}$ In this work the attosecond feature of the reflected radiation was emphasized, however, it was stated that the plasma behavior and the resulting attosecond pattern were not well understood.

In reality a laser pulse with a finite focal spot, cannot possess plane-wave behavior. A range of incidence angles appears along the interaction surface because of the transverse gradient of the pulse, coupled with significant excursions of relativistic electrons. This modifies the effective interaction surface contour.

Investigation of laser-plasma interaction in the relativistic $\lambda^{3}$ regime ( 5 fs laser pulses focused to $1-\lambda$ diameter spot) with two-dimensional (2D) and 3D particle-in-cell (PIC) simulations reveals that the radiation observed in reflection from overdense plasma is a train of attosecond pulses, with each pulse deflected to a specific angle, with a path different from the specular direction, and with a broadband spectral content. $^{7,8}$ This view is in agreement with experiments ${ }^{10-13}$ which indicate spectral broadening with higher intensity and spreading of radiation over larger angles. Matching plasma conditions with steplike profiles or exponential plasma gradients allows one to separate from the resulting radiation an isolated attosecond electromagnetic pulse. ${ }^{7,8}$ In addition, with increased incident intensity these attosecond pulses are scalable to still higher intensities, where shortening of the generated attosecond pulse durations leads to their greater focusability.

In overdense plasmas the laser pulse interacting with a target becomes a subwavelength driver for electrons because of the oscillating nature of the electromagnetic field. These 
laser-plasma interactions have been extensively investigated theoretically and experimentally. ${ }^{17}$ Recently evidence of ultrashort electron bunches has been found experimentally at the rear surface of thick solid targets. ${ }^{18}$ The bunched electrons were assigned to either vacuum or $v \times B$ heating mechanisms. ${ }^{19,20}$ These mechanisms are based on a 1D approach and the action of electric and magnetic parts in the Lorentz force on electrons that heat plasmas.

Analysis with 2D PIC simulations of laser-plasma interaction in the relativistic $\lambda^{3}$ regime reveals that dense attosecond electron bunches can be formed in vacuum. ${ }^{9}$ The attosecond pattern appears due to the joint action of incident and generated electromagnetic fields. In addition, the bunched electrons, escaping the target with relativistic velocities, compress the reflected radiation, generating the train of attosecond pulses. This illustrates a connection between the origin of attosecond electromagnetic pulses and bunched electrons, and their synchronized behavior.

In underdense plasmas $\left(\omega_{0}>\omega_{p}\right)$ with laser wakefield accelerators ${ }^{21}$ the typical duration of electron bunches is in the femtosecond time scale. ${ }^{22}$ In the regime of wave breaking, use of sharply spiked electron structure has been proposed for up-conversion and focusing of counterpropagating radiation. ${ }^{23}$ On the other hand, overdense plasmas driven in the relativistic $\lambda^{3}$ regime unconditionally emanate stable attosecond features due to superior spatial and temporal gradients.

\section{THE $\lambda^{3}$ REGIME AND PIC SIMULATIONS}

The idea to work in the relativistic $\lambda^{3}$ regime, with fewcycle laser pulses focused to $1-\lambda$ diameter spot, came from the fact that the maximal intensity can be achieved with available laser pulse energy. ${ }^{2}$ The properties of the highest gradients are enhanced in the $\lambda^{3}$ volume of laser-plasma interaction, when the laser pulse is incident on plasmas of critical or overcritical density. In this case the driver produces coherent relativistic motion of electrons, which efficiently interact with the electromagnetic environment.

The laser-plasma interaction in the $\lambda^{3}$ regime is by its nature extremely nonlinear. PIC simulations ${ }^{24}$ with fully relativistic self-consistent electromagnetic codes can provide insight to these interactions. The $\lambda^{3}$ regime, from first sight, demands 3D simulations. However taking into account a strong anisotropy of the interaction for linear polarized light, ${ }^{25}$ many details can be analyzed with $2 \mathrm{D}$ simulations when the electric field lies in the plane of incidence ( $p$ polarization). 2D simulations are much easier both in providing the necessary diagnostics and in the data representation. They are more agile when higher simulation resolution or parametric study is needed. Confirmation of the 2D behavior and specifics of 3D interactions can be studied then in the 3D space with reasonable simulation resolution.

Fully relativistic electromagnetic particle-in-cell code in 1D, 2D, and 3D versions working with Cartesian coordinates was used for simulation of relativistic laser-plasma interactions. Charge conservation was implemented and a secondorder form factor was used for charged particles. The plasma was considered to be collisionless and preionized. The laser (a)

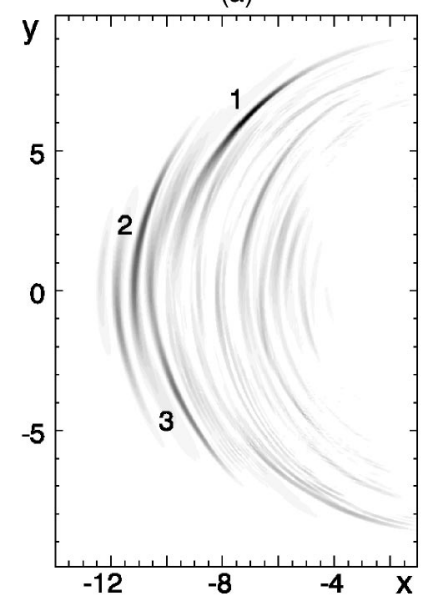

(b)

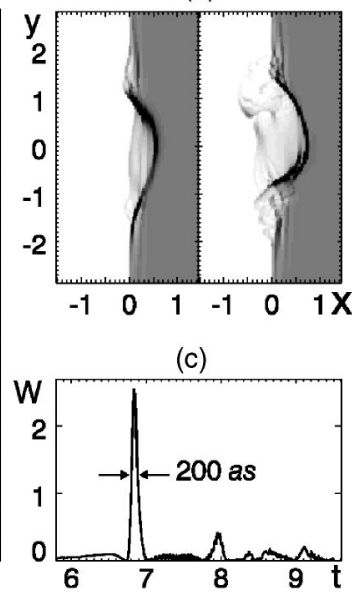

FIG. 1. Generation of attosecond electromagnetic pulses in a 2D PIC simulation. (a) The electromagnetic energy density of the reflected radiation $\left(W=E^{2}+B^{2}\right)$ at $t=11$. Numbers 1,2 , and 3 indicate the most intense pulses in the reflected radiation: 1 -with the highest intensity, 3-with the lowest intensity. (b) Snapshots of the electron density at instants $t=0.2, t=0.9$ when attosecond pulses: 3 and 1 are formed. (c) The time evolution of $W$ at the point $x=-3.5, y=3$. The arrows indicate the half-intensity level of an isolated pulse that contains $10 \%$ of the incident pulse energy. Simulation parameters: $a_{0}=3, \tau=5 \mathrm{fs}, n_{0}=1.5 n_{c r}$.

pulse was initiated at the left-hand boundary and propagated in vacuum along the $x$ axis toward the target. Periodic and absorbing boundary conditions were applied for electromagnetic fields and particles in transverse and longitudinal directions, respectively.

Studying attosecond phenomena with few-cycle incident pulses, we observed, that ion motion did not influence the fast electron dynamics. We choose $t=0$ to be the instant when the peak of the pulse envelope reaches the focal plane at $x=0$. In simulations spatial coordinates are measured in wavelengths, and time is measured in optical cycles.

\section{ISOLATED ATTOSECOND PULSES}

We begin the analysis of the relativistic $\lambda^{3}$ regime with 2D PIC simulations of $p$ polarized laser pulse incident on a plasma layer. ${ }^{7}$ Taking into account strong alternating electromagnetic fields of such a driver, we intend to separate a subcycle cut from the radiation. To reduce the number of parameters to be optimized we fix the pulse duration, focal spot size, and the amplitude in focus. We choose a pulse duration $\tau$ of 2 cycles (or $5 \mathrm{fs}$ for $\lambda=0.8 \mu \mathrm{m}$ ), a 1- $\lambda$ diameter focal spot, a dimensionless amplitude in focus $a_{0}=3$, and a Gaussian profile and cosinelike temporal field (i.e., with 1 maximum), such as are experimentally accessible. For simplicity, we choose steplike plasma profile and focus the laser pulse normal to the plasma layer (the average propagation vector is along $x$ axis, and the plasma boundary is along $y$ axis).

Varying the plasma density, under these conditions we find the optimal value $n_{0}=1.5 n_{c r}$ (here $n_{c r}=m_{e} \omega_{0}^{2} / 4 \pi e^{2}$ ), where the radiation is efficiently deflected by the plasma surface. The result of this simulation is shown in Fig. 1. On the plot of the electromagnetic energy density in Fig. 1(a) we observe that reflected radiation is deflected to different 


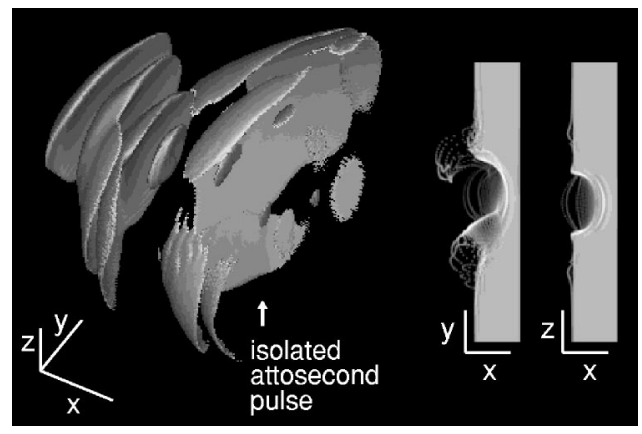

FIG. 2. Generation of attosecond electromagnetic pulses in a 3D PIC simulation. Isosurface of the electromagnetic energy density demonstrates efficient generation of isolated attosecond pulse (left). Electron density slices show anisotropic electron activity at the time of attosecond pulse generation (right). Simulation parameters: $a_{0}=3, \tau=5 \mathrm{fs}, n_{0}=1.5 n_{c r}$.

angles. In addition, the oscillating electric field breaks the lateral symmetry, and subcycle pulses are deflected to one side or the other of the specular direction. We indicate the three most prominent pulses and find that the strongest pulse has a larger deflection angle $\sim 40^{\circ}$. A similar plot marking the half-intensity level reveals that the strongest pulse is isolated, and has a divergence of $\sim 20^{\circ}$.

Observing the electron density plots in Fig. 1(b), we find that electrons move with relativistic velocities, creating different reflection conditions at each half cycle. These fast electrons move coherently and provide a Doppler time transformation for the reflected radiation. On the plot of the electromagnetic energy density in Fig. 1(c) at a point along the maximal value path of the strongest pulse we estimate the pulse duration to be 200 as. Within its half-intensity isocontour the pulse contains $\sim 10 \%$ of incident optical pulse energy.

The analytical model of a relativistically driven charged layer ${ }^{7}$ confirms that an oscillating electric field at oblique incidence is sufficient to provide the pulse compression. Depending on the electric field phase the charged sheet moves from or toward the reflected radiation, forming stretched background radiation or prominent spikes. Indeed, both features are observed in the reflected radiation in Fig. 1(c). Consequently, the self-induced relativistic motion of charges in the $\lambda^{3}$ regime directly generates isolated attosecond pulses by reflection, deflection, and compression.

3D simulations performed for the same laser-plasma parameters ${ }^{8}$ show substantially the same effect including efficient isolated attosecond pulse formation [Fig. 2]. Results similar to the 2D behavior are observed when viewing the $(x, y)$ plane. While in the $(x, z)$ plane, a significantly higher degree of symmetry is present on both the plot at the halfintensity level of the reflected radiation and on the plot of electron density. Also, the divergence of the isolated attosecond pulse is larger in the $(x, z)$ plane. Thus the knowledge of the dominant features responsible for the formation of isolated attosecond pulses understood by the results of 2D simulations is confirmed and extended into full 3D space.

To be close to realistic plasma conditions, we performed 2D simulations for different angles of incidence and exponential plasma profiles. ${ }^{8}$ Again efficient generation of iso- lated attosecond pulses is observed. We find the most effective attosecond pulse formation and isolation for a cosinelike incident laser pulse, where the carrier phase is chosen so that the peak electric field is oriented toward the specular reflection. This results in the strongest compressed pulses. Also, they propagate not in the specular direction, but between the specular direction and the surface of the target.

In general the path of compressed attosecond pulses is not found in the specular direction, but to one side or the other. We might associate this with the fact that the fast motion of the surface that causes compression must also be skewed out of symmetry by the relativistic effect that pushes more at the peak of each optical half-cycle, bending the reflection off the optical axis.

To scale this mechanism of attosecond pulse generation to higher intensities, it is necessary to keep similar slopes in electron density profiles. To do so, the plasma density should be scaled linearly with the driving field strength, maintaining the same balance between charge separation forces and forces arising from the incident fields. With higher field strength, electrons also move faster and the resulting Doppler compression leads to the formation of still shorter pulses.

Using 2D PIC simulations, we find that the ratio of $a_{0} / n_{0} \simeq 2$ is optimal for the production of isolated attosecond pulses. Boosted-frame 1D PIC simulations are capable of modeling oblique incidence only over a limited range of amplitude, because reflected light is bound to propagate only in the specular direction. Nevertheless, we find that attosecond pulse duration produced follow the dependence $\propto a_{0}^{-1}$ for the ratio $a_{0} / n_{0}=2$ in the range $3 \leqslant a_{0} \leqslant 20 .^{8}$

\section{SELF-INDUCED FOCUSING OF LIGHT}

In a simple view, attosecond electromagnetic pulses, emerging from a target which is curved by the laser pulse pressure, can be simultaneously focused to a much smaller volume, providing higher intensity in focus. To demonstrate this potential effect, we performed preliminary 2D simulations for the stated above laser pulse parameters and for the steplike plasma profile.

Evaluation of the maximal value of the electromagnetic energy density in time leads to the optimal density value $n_{0}=4 n_{c r}$, for which the strongest generated attosecond pulse gives the maximal intensity in its focus, after emerging from the target. With variation of the plasma density, the "focusing optics" and the attosecond pulse duration are changed. The former depends mainly on the ratio $a_{0} / n_{0}$, and the latter depends on $a_{0}$ and $a_{0} / n_{0}$.

For the parameters chosen in 2D simulations we perform a $3 \mathrm{D}$ simulation in the box of $(4 \lambda)^{3}$ volume, with 100 grid cells per $\lambda$, and 8 particles per cell. This simulation, which is global in its representation, encompasses in the volume of a few $\lambda^{3}$, has sufficient resolution, and is accessible with moderate computer facilities.

In the 3D simulation we find that the focal intensity of attosecond electromagnetic pulse increases $\sim 15$ times in respect to the focal intensity of the incident laser pulse in vacuum. Figure 3 shows the strongest attosecond pulse both in focus, when it has its maximal intensity, and after the 


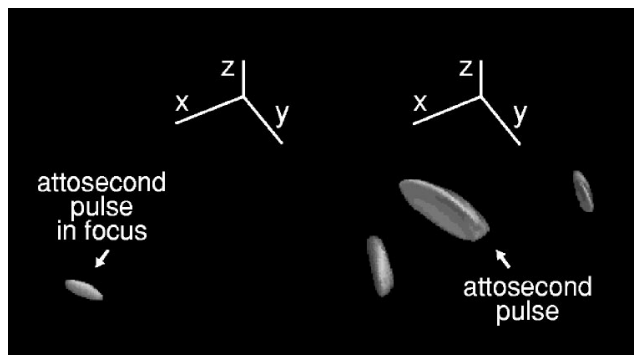

FIG. 3. Self-induced focusing in a 3D PIC simulation. Isosurface of the electromagnetic energy density at the half-intensity level in (left) and after focus (right). Simulation parameters: $a_{0}=3, \tau=5 \mathrm{fs}, n_{0}=4 n_{c r}$.

focus. The location of the focal volume is in vacuum at a distance of $0.6 \lambda$ from the original plasma boundary. And again the deflection of attosecond pulses is observed in the $(x, y)$ plane. The focal volume is also tilted at a larger angle, indicating the complexity and nonuniformity of attosecond pulse generation along the radiating charged surface. The attosecond pulse in focus encompasses a volume of $\sim 10^{-3} \lambda^{3}$ ! within its half-intensity isosurface; as in previous 3D simulation, it has anisotropic structure both in and after focus.

In simulations, the induced focusing of a loosely focused laser pulse into more narrow beam has been observed in reflection from a thin foil. ${ }^{26}$ Here we show that the focal volume of an attosecond electromagnetic pulse generated in reflection from a plasma surface can be very small fractions of the $\lambda^{3}$ volume, with a commensurate increase of focal intensity. Indeed, this effect of self-induced focusing of light is similar to the self-focusing observed in underdense plasma, ${ }^{27,28}$ when the laser pulse changes a refractive index and converges the wave front producing higher intensity.

\section{ATTOSECOND ELECTRON BUNCHES}

Analyzing attosecond pulse generation at different angles, we observe on electron density plots [Fig. 1(b)] that electrons move in thin dense sheets with relativistic velocities, reflecting, deflecting, and compressing the radiation. Fast electron sheets can exit plasmas passing into vacuum, but the incident radiation from subsequent cycles and the charge separation effect can dramatically change their quality and even destroy them. This is possible to overcome by increasing the angle of incidence, when the electrons having escaped into vacuum are supported by the joint action of the incident and reflected wave.

We demonstrate the effect of electron ejection and formation of attosecond electron bunches in vacuum ${ }^{8,9}$ with $2 \mathrm{D}$ PIC simulation for a $15 \mathrm{fs} p$ polarized laser pulse focused to a $1-\lambda$ diameter spot at $70^{\circ}$ incidence on a plasma target of density of $25 n_{c r}$. The dimensionless amplitude in focus is $a_{0}=10$.

In Fig. 4(a) we observe that the $e E$ and $e v \times B$ forces of the electromagnetic field create a strong grazing pressure on the plasma, enhanced by interference between the incident and reflected waves. Electrons from the skin layer are pushed into the plasma. Simultaneously, due to charge separation effects, counterstreaming of electrons occurs. Some counter- (a)

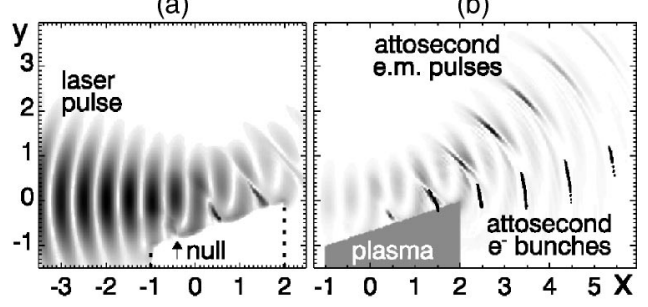

(c)

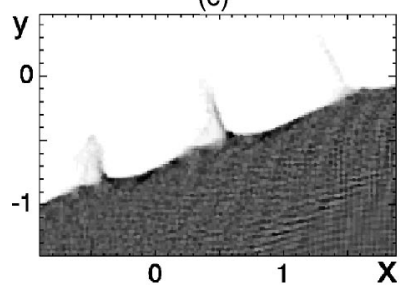

(d)

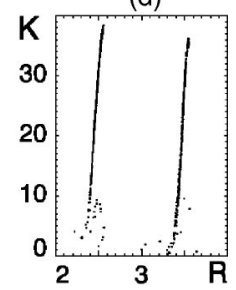

FIG. 4. Trains of attosecond electron bunches and attosecond electromagnetic pulses formed at oblique incidence on a short target. Electromagnetic energy density (gray scale): (a) $W^{1 / 2}$ at $t=-3$ and (b) $W$ at $t=3$. In (a) the arrow indicates an electromagnetic field null and in (b) overlapped black dots indicate 25-30 MeV electrons. (c) Electron jets emerging from the target on the electron density plot at $t=-3$. (d) Kinetic energy (in MeV) of bunched electrons in an arc projection at $t=3$. Simulation parameters: $a_{0}$ $=10, \tau=15 \mathrm{fs}, n_{0}=25 n_{c r}$.

streaming electrons are ejected from the plasma through narrow, localized nulls in the electromagnetic field, into a sweeping field which, having reversed, supports their ejection and further acceleration [Figs. 4(a) and 4(c)].

Escaping the plasma with relativistic velocities, these electrons compress the reflected radiation and form attosecond electromagnetic pulses while maintaining their own attosecond bunched structure [Figs. 4(b) and 4(d)]. The trains of attosecond electromagnetic pulses and electron bunches are mutually synchronized, having been formed cooperatively.

Estimating the number of particles in the strongest bunches, we find that they contain $\sim 2 \times 10^{8}$ electrons for these laser-plasma parameters. The energy distribution in each bunch is chirped [Fig. 4(d)], and above $10 \mathrm{MeV}$ the bunch thickness is $\lambda / 7$. As the maximal energy in the bunch depends on the maximal amplitude in the corresponding cycle, by shaping the laser pulse it is possible to generate a single attosecond electron bunch.

In evaluating the efficiency of the attosecond features generated we find that $\sim 15 \%$ of an incident peak cycle has been converted to a corresponding attosecond pulse (integrating within the $1 / e$ isointensity contour). Also, the bunched electrons have gained $\sim 15 \%$ of the incident pulse energy. These data demonstrate the specific mechanism of the energy conversion: the incident pulse energy is efficiently converted to the energy of attosecond electromagnetic pulses via interaction with relativistic electron bunches.

\section{DISCUSSION}

Beginning with efficient use of focused laser energy to achieve high field and interacting with overdense plasma we find an efficient route to attosecond physics. In general, short pulse formation occurs whenever relativistic pulses interact 
with near-critical or overcritical plasmas, but, more responsive plasmas act more efficiently. We find that scalable isolated attosecond pulses can be efficiently generated and focused to significantly higher intensities. Also, dense attosecond electron bunches can be produced in synchronism with attosecond electromagnetic pulses. Utilizing this $\lambda^{3}$ concept we also find that it is possible to make a compact 3D simulation that fully encompasses the problem.

An investigation of attosecond physics arising in strongly driven overdense plasmas shows the relativistic $\lambda^{3}$ regime to be fertile ground for the efficient generation of attosecond phenomena. Indeed, the $\lambda^{3}$ regime lends itself to isolation of any phenomenon arising in the laser-plasma interactions. The fact that electromagnetic pulses are found to be isolated spatially should also make experiments easier to evaluate.

What connection does this have with physical observations and what potential does it offer? In light of these discoveries first experiments aimed at confirming attosecond pulse durations in the relativistic $\lambda^{3}$ regime are in progress. ${ }^{29,30}$ Using $0.8 \mu \mathrm{m}, 30 \mathrm{fs}$ linearly polarized laser pulses focused to $1.2 \mu \mathrm{m}$ diameter spot, splitting of reflected radiation into two lobes is observed. ${ }^{30}$ We interpret this as an indication that radiation trains are emerging from the two sides of the relativistically driven plasma surface. Also, the observed divergence of the resulting radiation in the plane of the electric field is narrower than in the perpendicular plane. These observations are both in agreement with the results of the PIC simulations discussed here.

If the phenomena discussed in this paper are realized experimentally, they offer the potential for the generation of extreme fields and efficient attosecond x-ray generation. Under the influence of intensities available using current laser technology the potential exists to efficiently generate pulses with several-attosecond duration and focusability to a few nanometers. Such intense fields should be strong enough to extract significant numbers of electron-positron pairs from vacuum.

The dense attosecond electron bunches have the ability to scatter counterpropagating electromagnetic radiation with a frequency upshift of $4 \gamma^{2}$. Analysis of the influence of the bunch thickness $d$ on the scattering efficiency with respect to the wave vector $k$ leads to the conclusion that near unity conversion efficiency may be obtained under the condition $k d \sim 1 / 2 \gamma^{2},{ }^{9}$, provided a sufficient number of electrons is included in the bunch. The chirped energy distribution observed in simulations might be exploited to obtain high scattering efficiency from a lower charge density, as discussed in Ref. 9. Implementing this scattering would be a means of generating extremely bright attosecond x-ray pulses.

In short, the efficiency derived from working with overdense plasmas in the relativistic $\lambda^{3}$ regime enables a new optoelectronics based on relativistic effects and operating in the attosecond domain.

\section{ACKNOWLEDGMENTS}

This work was supported by the NSF (Grant No. 0114336) and the ARO (Grant Nos. DAAD19-03-1-0287 and DAAD19-03-1-0316). Simulations have been performed in the Center for Advanced Computing, University of Michigan.

${ }^{1}$ G. A. Mourou, P. J. C. Barty, and M. D. Perry, Phys. Today 51(1), 22 (1998).

${ }^{2}$ G. Mourou, Z. Chang, A. Maksimchuk, J. Nees, S. Bulanov, V. Bychenkov, T. Esirkepov, N. Naumova, F. Pegoraro, and H. Ruhl, Plasma Phys. Rep. 28, 12 (2002).

${ }^{3}$ S. Bahk, P. Rousseau, T. Planchon, V. Chvykov, G. Kalintchenko, A. Maksimchuk, G. Mourou, and V. Yanovsky, Opt. Lett. 29, 2837 (2004).

${ }^{4}$ T. Tajima and G. Mourou, Phys. Rev. ST Accel. Beams 5, 031301 (2002).

${ }^{5}$ S. V. Bulanov, F. Califano, G. I. Dudnikova et al., in Reviews of Plasma Physics, edited by V. D. Shafranov (Kluwer Academic, New York, 2001), Vol. 22, p. 227.

${ }^{6}$ G. Mourou, T. Tajima, and S. Bulanov, Rev. Mod. Phys. (in press).

${ }^{7}$ N. M. Naumova, J. A. Nees, I. V. Sokolov, B. Hou, and G. A. Mourou, Phys. Rev. Lett. 92, 063902 (2004).

${ }^{8}$ J. Nees, N. Naumova, E. Power et al., J. Mod. Opt. 52, 305 (2005).

${ }^{9}$ N. Naumova, I. Sokolov, J. Nees, A. Maksimchuk, V. Yanovsky, and G. Mourou, Phys. Rev. Lett. 93, 195003 (2004).

${ }^{10}$ R. L. Carman, C. K. Rhodes, and R. F. Benjamin, Phys. Rev. A 24, 2649 (1981).

${ }^{11}$ P. A. Norreys, M. Zepf, S. Moustaizis et al., Phys. Rev. Lett. 76, 1832 (1996).

${ }^{12}$ A. Tarasevitch, A. Orisch, D. von der Linde, Ph. Balcou, G. Rey, J.-P. Chambaret, U. Teubner, D. Klöpfel, and W. Theobald, Phys. Rev. A 62, 023816 (2000).

${ }^{13}$ U. Teubner, G. Pretzler, Th. Schlegel, K. Eidmann, E. Förster, and K. Witte, Phys. Rev. A 67, 013816 (2003).

${ }^{14}$ S. V. Bulanov, N. M. Naumova, and F. Pegoraro, Phys. Plasmas 1, 745 (1994).

${ }^{15}$ R. Lichters, J. Meyer-ter-Vehn, and A. M. Pukhov, Phys. Plasmas 3, 3425 (1996).

${ }^{16}$ L. Plaja, L. Roso, K. Rzążewski, and M. Lewenstein, J. Opt. Soc. Am. B 15, 1904 (1998)

${ }^{17}$ S. C. Wilks and W. L. Kruer, IEEE J. Quantum Electron. 33, 1954 (1997), and references therein.

${ }^{18}$ S. Baton, J. Santos, F. Amiranoff et al., Phys. Rev. Lett. 91, 105001 (2003).

${ }^{19}$ F. Brunel, Phys. Rev. Lett. 59, 52 (1987).

${ }^{20}$ W. Kruer and K. Estabrook, Phys. Fluids 28, 430 (1985).

${ }^{21}$ E. Esarey, P. Sprangle, J. Krall, and A. Ting, IEEE Trans. Plasma Sci. 24, 252 (1996), and references therein.

${ }^{22}$ S. Fritzler, K. Ta Phuoc, V. Malka, A. Rousse, and E. Lefebvre, Appl. Phys. Lett. 83, 3888 (2003)

${ }^{23}$ S. V. Bulanov, T. Esirkepov, and T. Tajima, Phys. Rev. Lett. 91, 085001 (2003).

${ }^{24}$ C. K. Birdsall and A. B. Langdon, Plasma Physics via Computer Simulation (McGraw-Hill, New York, 1985).

${ }^{25}$ N. M. Naumova, S. V. Bulanov, K. Nishihara, T. Zh. Esirkepov, and F. Pegoraro, Phys. Rev. E 65, 045402 (2002).

${ }^{26}$ V. A. Vshivkov, N. M. Naumova, F. Pegoraro, and S. V. Bulanov, Phys. Plasmas 5, 2752 (1998).

${ }^{27}$ A. B. Borisov, A. V. Borovskiy, V. V. Korobkin et al., Phys. Rev. Lett. 68, 2309 (1992).

${ }^{28}$ P. Monot, T. Auguste, P. Gibbon, F. Jakober, G. Mainfray, A. Dulieu, M. Louis-Jacquet, G. Malka, and J. L. Miquel, Phys. Rev. Lett. 74, 2953 (1995).

${ }^{29}$ E. Power, N. Naumova, J. Nees, A. Maksimchuk, V. Yanovsky, I. Sokolov, K. Hong, T. Matsuoka, B. Hou, and G. Mourou, Bull. Am. Phys. Soc. 49(8), 104 (2004).

${ }^{30}$ E. P. Power, J. A. Nees, N. M. Naumova, K.-H. Hong, T. Matsuoka, V. P. Yanovsky, B. Hou, G. A. Mourou, and I. V. Sokolov, "Experimental observations of the relativistic deflection of light," Conference on Lasers and Electro-Optics (CLEO/QELS 2005, Baltimore) (to be published). 